Louisiana State University

LSU Digital Commons

$1-1-2014$

\title{
Cryptic diversity hides host and habitat specialization in a gorgonian-algal symbiosis
}

\author{
Carlos Prada \\ Louisiana State University \\ Shelby E. Mcllroy \\ University at Buffalo, The State University of New York \\ Diana M. Beltrán \\ University of Puerto Rico-Mayaguez \\ Daniel J. Valint \\ University at Buffalo, The State University of New York \\ Scott A. Ford \\ University at Buffalo, The State University of New York
}

See next page for additional authors

Follow this and additional works at: https://digitalcommons.Isu.edu/biosci_pubs

\section{Recommended Citation \\ Prada, C., Mcllroy, S., Beltrán, D., Valint, D., Ford, S., Hellberg, M., \& Coffroth, M. (2014). Cryptic diversity hides host and habitat specialization in a gorgonian-algal symbiosis. Molecular Ecology, 23 (13), 3330-3340. https://doi.org/10.1111/mec.12808}




\section{Authors}

Carlos Prada, Shelby E. Mcllroy, Diana M. Beltrán, Daniel J. Valint, Scott A. Ford, Michael E. Hellberg, and Mary Alice Coffroth 


\title{
Cryptic diversity hides host and habitat specialization in a gorgonian-algal symbiosis
}

\author{
CARLOS PRADA,${ }^{* 1}$ SHELBY E. MCILROY,$\dagger^{1}$ DIANA M. BELTRÁN, + DANIEL J. VALINT, $\uparrow$ SCOTT \\ A. FORD,$\uparrow$ MICHAEL E. HELLBERG* and MARY ALICE COFFROTH† \\ *Department of Biological Sciences, Louisiana State University, Baton Rouge, LA, 70803, USA, †Department of Geology and \\ Graduate Program in Evolution, Ecology and Behavior, State University of New York at Buffalo, Buffalo, NY, 14260, USA, \\ †Department of Marine Sciences, University of Puerto Rico, Mayagüez, PR, 00667, USA
}

\begin{abstract}
Shallow water anthozoans, the major builders of modern coral reefs, enhance their metabolic and calcification rates with algal symbionts. Controversy exists over whether these anthozoan-algae associations are flexible over the lifetimes of individual hosts, promoting acclimative plasticity, or are closely linked, such that hosts and symbionts co-evolve across generations. Given the diversity of algal symbionts and the morphological plasticity of many host species, cryptic variation within either partner could potentially confound studies of anthozoan-algal associations. Here, we used ribosomal, organelle and nuclear sequences, along with microsatellite variation, to study the relationship between lineages of a common Caribbean gorgonian and its algal symbionts. The gorgonian Eunicea flexuosa is a broadcast spawner, composed of two recently diverged, genetically distinct lineages largely segregated by depth. We sampled colonies of the two lineages across depth gradients at three Caribbean locations. We find that each host lineage is associated with a unique Symbiodinium B1/184 phylotype. This relationship between host and symbiont is maintained when host colonies are reciprocally transplanted, although cases of within phylotype switching were also observed. Even when the phylotypes of both partners are present at intermediate depths, the specificity between host and symbiont lineages remained absolute. Unrecognized cryptic diversity may mask host-symbiont specificity and change the inference of evolutionary processes in mutualistic associations. Symbiotic specificity thus likely contributes to the ecological divergence of the two partners, generating species diversity within coral reefs.
\end{abstract}

Keywords: broadcast spawner, co-evolution, depth, ecological speciation, Symbiodinium

Received 14 February 2014; revision received 21 May 2014; accepted 21 May 2014

\section{Introduction}

An obligate association between cnidarians and unicellular algae of the genus Symbiodinium forms the foundation of coral reefs, the most diverse marine ecosystem. In these nutrient-poor environments, algae provide organic compounds and energy to their coral hosts, while the coral provides recycled inorganic nutrients and access to a stable light environment for the alga

Correspondence: Carlos Prada, Fax: 225578 2597;

E-mail: cprada1@lsu.edu

${ }^{1}$ Authors contribute equally to this work.
(Muscatine et al. 1981, 1984; Colombo-Pallotta et al. 2010). This endosymbiosis is physiologically controlled by both partners (Muscatine et al. 1983; Davy et al. 2012). At the genomic level, the coral's functional genome is uniquely shaped by different Symbiodinium species (DeSalvo et al. 2010). At the extreme, incompatible symbionts activate immune responses in their hosts, even in coral species that associate with multiple distantly related phylotypes (Voolstra et al. 2009). The synchronized efforts of both partners affect their growth and thermal tolerance (Little et al. 2004; Abrego et al. 2008), so that Symbiodinium can facilitate coral acclimation to heterogeneous environments (Chen et al. 2005; 
Sampayo et al. 2007) and even to warmer oceans (Rowan et al. 1997; Baker 1999; Rowan 2004; Berkelmans \& van Oppen 2006; Sampayo et al. 2008).

Given the functional and phylogenetic diversity within Symbiodinium, corals may acclimate through symbiont acquisition. Once known as a single species, nine major clades of Symbiodinium are now recognized, with intercladal genetic distances at rDNA markers that far exceed those among bird families (Rowan \& Powers 1992; Rowan \& Knowlton 1995; Coffroth \& Santos 2005; LaJeunesse et al. 2010; Pochon \& Gates 2010). Lineages within these clades are often segregated by habitat (usually depth) (Rowan \& Knowlton 1995; Sampayo et al. 2007) and geography (LaJeunesse et al. 2010). Corals can exploit functional differences among these Symbiodinium lineages either via facultative associations that may vary over a host's lifetime or species-specific coupling, which results in co-evolution of corals and dinoflagellates.

At deep evolutionary scales (i.e. family level), corals may associate with more than one highly divergent lineage of dinoflagellates in the genus Symbiodinium, with some coral species harbouring multiple symbiont phylotypes within a single colony (Rowan \& Knowlton 1995; Little et al. 2004). At first glance, then, corals may seem flexible in their associations with Symbiodinium and able to interact with any symbiont type. The uptake of multiple genotypes of Symbiodinium is notorious early in coral development, when many Symbiodinium species can be found within a developing polyp (Poland et al. 2013). However, while background or transient symbiont phylotypes may exist (Mieog et al. 2007; LaJeunesse et al. 2009; Silverstein et al. 2012), most adult anthozoan colonies host a single-dominant algal type (Goulet \& Coffroth 2003; Thornhill et al. 2009; Finney et al. 2010; Pettay et al. 2011), opening the possibility for co-evolution between partners (LaJeunesse et al. 2004; LaJeunesse 2005; Finney et al. 2010; Thornhill et al. 2013, 2014). At microevolutionary scales, symbiont types may associate with individual anthozoan species and even intraspecific phylogeographical lineages (Santos et al. 2003b; LaJeunesse 2005; Goulet 2006; Finney et al. 2010; Pinzon \& LaJeunesse 2011). These close evolutionary associations are particularly pronounced in shallow water Caribbean gorgonians, which associate almost exclusively with Symbiodinium in clade B (LaJeunesse 2002; Santos et al. 2004; Goulet et al. 2008). Recent work has verified that Clade B (as other clades) contains many cryptic species, with a high degree of host-symbiont specificity (Finney et al. 2010).

Fine scale genetic markers, such microsatellites, provide the ability to test for co-evolution at the population level (Santos et al. 2003b, 2004). Genetic variation and structure in host populations have been correlated with host reproductive traits (i.e., brooding vs. spawning, larval duration, (Kahng et al. 2011)), while the major factors that affect Symbiodinium distribution include limited dispersal (Santos et al. 2003b; Magalon et al. 2006; Thornhill et al. 2009; LaJeunesse et al. 2010) and, in particular, light environment (Rowan et al. 1997; Finney et al. 2010). Selection pressures that affect the distribution of one or both partners can shape co-evolution; however, reciprocal evolutionary change can only be detected when the genetic structure of both partners are examined across relevant spatial scales.

Eunicea flexuosa is a broadcast spawning gorgonian with larvae that acquire their symbionts horizontally from the water column (Beiring \& Lasker 2000; Coffroth et al. 2001; Kim et al. 2004). Thus, relative to brooding corals with vertical transmission, E. flexuosa maintains the potential to shuffle its symbionts across generations. A microsatellite-based analysis of E. flexuosa and Symbiodinium population structure by Wirshing et al. (2013) found that, contrary to previous studies in Caribbean gorgonians (Santos et al. 2003b; Coffroth \& Santos 2005; Andras et al. 2009; Kirk et al. 2009), the genetic structure of host and symbiont populations were not correlated. However, the sampling design of this study did not consider depth as a potential structuring force. Cryptic host diversity and habitat (depth) thus offer unexplored alternative explanations for the genetic variation of Symbiodinium found in E. flexuosa.

Here, we elucidate variation in Symbiodinium associated with the recently diverged shallow and deep lineages of E. flexuosa using four different types of molecular markers. Eunicea flexuosa provides an excellent system for disentangling the roles of host specificity, habitat and geography in partitioning Symbiodinium diversity because: (i) E. flexuosa is composed of a sympatric pair of recently diverged shallow and deep lineages (Prada et al. 2008; Prada \& Hellberg 2013); (ii) these host lineages occasionally co-occur at intermediate depths (Prada \& Hellberg 2013); and (iii) E. flexuosa lineages are widespread across the Caribbean and the shallow/deep distribution varies geographically (Bayer 1961; Prada \& Hellberg 2013).

We first analysed Symbiodinium variation at higher taxonomic levels using ribosomal ITS2 and chloroplast sequences, along with nuclear sequences to elucidate within clade B variation. We then used microsatellites to resolve population subdivision within Symbiodinium lineages at geographical scales. We also tested the stability of the host-symbiont relationships with a reciprocal transplant experiment. If coral-algal associations are mainly driven by host species identity (Finney et al. 2010; LaJeunesse et al. 2010), then colonies in reciprocal transplants will rarely switch symbiont types, and host 
colonies will remain true to symbiont types, even when occurring outside their usual depth.

\section{Materials and methods}

\section{Sampling and sequence data}

Colonies were sampled at three locations: Puerto Rico, Bahamas and Curaçao [for full descriptions of these locations see (Prada \& Hellberg 2013)]. These locations span regions where the distributions of depth-segregated cryptic species within Eunicea flexuosa have been previously investigated (Prada \& Hellberg 2013). At each location, we sampled adult colonies $(>50 \mathrm{~cm}$ in height) at two depths, as earlier work suggested that deep $(>20 \mathrm{~m})$ and shallow $(<5 \mathrm{~m})$ populations are genetically differentiated across the Caribbean (Prada et al. 2008; Prada \& Hellberg 2013). At each depth, we collected tissue from between 16 and 38 colonies. To avoid sampling clones, collected colonies were at least $5 \mathrm{~m}$ apart. Multilocus genotypes confirmed that all colonies came from unique genets.

To disentangle the roles of host species identity and depth, we also sampled 40 colonies from intermediate depths (12-15 m) where the E. flexuosa lineages cooccur. In Puerto Rico, we also reciprocally transplanted coral colonies to native and foreign depths (see above; 38 per depth, 152 total fragments) and resampled them after 18 months. Fitness across depths for these colonies was assessed previously (Prada \& Hellberg 2013). We preserved all samples in $95 \%$ ethanol at $-20{ }^{\circ} \mathrm{C}$. We extracted genomic DNA from these samples using either a 2X CTAB method (Coffroth et al. 1992) or a QIAGEN DNeasy Kit following the manufacture's protocols.

Host genotypes were scored for one mtDNA marker, msh, and three nuclear markers: inositol-3-phosphatase $(i 3 p)$, elongation factor 1 alpha (ef1a) and calcium transporter 2 (ct2). These multilocus genotypes have been used previously to discern the two depth-based cryptic E. flexuosa lineages (Prada \& Hellberg 2013).

To determine which Symbiodinium clade was found in E. flexuosa, we amplified the ribosomal ITS2 and the hypervariable region of the chloroplast 23s-rDNA following earlier protocols [(LaJeunesse 2002) for ITS2; (Santos et al. 2003a) for cp23s-rDNA]. We sequenced 224 bases of the ITS2 (see below). The cp23s-rDNA amplicons were sized in polyacrylamide gels using a metric ruler with DNA ladders as size references, and alleles were named based on established clade and fragment length nomenclature (Santos et al. 2003a).

To identify cryptic lineages within Symbiodinium B1/ 184, the predominant symbiont type, we used DNA sequences from two chloroplast genes and one nuclear locus. We amplified and sequenced samples for the two chloroplast markers following previous protocols [(Moore et al. 2003) for psbA; (Santos et al. 2002) for cp23s-rDNA]. We also sequenced a subset of samples (42) from all populations (three locations and two depths) using the flanking region of the microsatellite B7SYM15, following previous procedures (Pettay \& LaJeunesse 2007). Amplicons were directly sequenced in both directions (except B7SYM15, forward only) in an ABI 3100 using BigDye chemistry v 3.1 and both amplification primers. Symbiodinium is haploid, so no allele phasing was required. We edited and assembled all sequences using Geneious v4.5.5 (Drummond et al. 2009).

To further characterize genetic variation across sampling location in Symbiodinium B1/184, we genotyped all colonies at four microsatellite markers: B7SYM15, B7SYM34, B7SYM36 (Pettay \& LaJeunesse 2007) and GV2 100 (Andras et al. 2009). To test for host-symbiont associations following a change in environment, we genotyped post-transplanted colonies at the B7SYM15, B7SYM34 and B7SYM36 loci. Primers and PCR conditions for these loci were carried out according to conditions in previous studies (Pettay \& LaJeunesse 2007; Andras et al. 2009). Fragments were amplified with fluorescently labelled primers and visualized on a $7 \%$ polyacrylamide gel in a LI-COR NEN ${ }^{\circledR}$ Global IR2 DNA Sequencer (Santos et al. 2003a,b). Microsatellites were scored by eye relative to 50-350 bp size standards (LICOR Biotechnology Division).

\section{Data analysis}

To identify the patterns of associations between Symbiodinium within clade variants and Eunicea flexuosa, we built a phylogeny based on the ribosomal ITS2. To root the genealogy, we included sequence data from a recent description for Symbiodinium B1 (LaJeunesse et al. 2012) (AF333510, AF333511 and AF333512) and also from the widely used culture 704 (JN558059). We estimated the model of molecular evolution using MRAIC v. 1.4.5 (Nylander 2004). We used Bayesian analyses to estimate each gene tree (Mr. Bayes v. 3.1) and estimated nodal support using posterior probabilities (Huelsenbeck \& Ronquist 2001). We also constructed parsimony haplotype networks for the chloroplast markers cp23s and psbA and the nuclear B7SYM15 using the Templeton et al. (1992) algorithm implemented in TCS 1.21 (Clement et al. 2000). Each network was constructed with a confidence level set at 95\% and excluding gaps.

Once genealogical patterns had been uncovered, we analysed hierarchical genetic subdivision within the two major Symbiodinium lineages using microsatellite data. To apportion genetic subdivision among host 
genotype, depth and geography, we used Analysis of Molecular Variance (AMOVA) as implemented in GENODIVE (Meirmans \& Van Tienderen 2004). We performed 100000 permutations using standard F-statistics. We defined populations by host's genotype, depth and geography. To further characterize geographical differentiation within the Symbiodinium group designated B1/B184, we used a Bayesian clustering approach implemented by STRUCture (Pritchard et al. 2000). Because multiple algal genotypes were found in 44 of the host colonies, we analysed the data in two ways. We first generated all the multilocus combinations that could possibly be generated for each colony (full data set). Alternatively, we eliminated all colonies with ambiguous genotypes (short data set).

We used the admixture model in STRUCTURE without information of the origin of each individual. For each STRUCTURE analysis, we set up a burn-in of one million steps followed by 5 million iterations and 10 replicates per run. We ran STRUCTURE using a variety of K's (number of inferred populations) from the minimum (1) to the maximum (6) and then used the Evanno method (Evanno et al. 2005) as implemented in STRUCTURE HARVESTER (Earl \& vonHoldt 2011) to infer the number of populations $\left(\mathrm{K}^{\prime} \mathrm{s}\right)$ present in the data set. We processed replicates with best $\mathrm{K}$ (either 2 or 5) in CLuMMP (Jakobsson \& Rosenberg 2007) using the default parameters. To generate figures, we used Distruct 1.1 (Rosenberg 2004). To test for host-symbiont associations between depths, we ran STRUCTURE assuming the most likely $\mathrm{K}$ (=2) for samples collected in Puerto Rico.

\section{Results}

Shallow and deep lineages of Eunicea flexuosa associate with Symbiodinium B1/184

Both ITS2 and chloroplast data suggest that all Eunicea flexuosa colonies harbour Symbiodinium phylotype B1/ B184, regardless of the host's genotype, depth or geography (Fig. 1). Of the 183 colonies sampled, no colony contained any Symbiodinium from other clades or any other clade B types. We re-covered a single ITS2 haplotype for all individuals, but multiple haplotypes for cp23s, psbA and SYM15 (Fig. 2). Genetic variation within these three loci revealed differences in algal composition between host colonies sampled in shallow and deep habitats (Fig. 2). Three haplotypes were re-covered from analysis of SYM15. These divided into two distinct lineages, which sorted by depth and matched the shallow and deep host lineages. $p s b A$ also had three haplotypes: one found solely in shallow hosts, another solely in deep hosts, with a third found mainly in deep hosts but also in shallow colonies from the Bahamas. cp23s

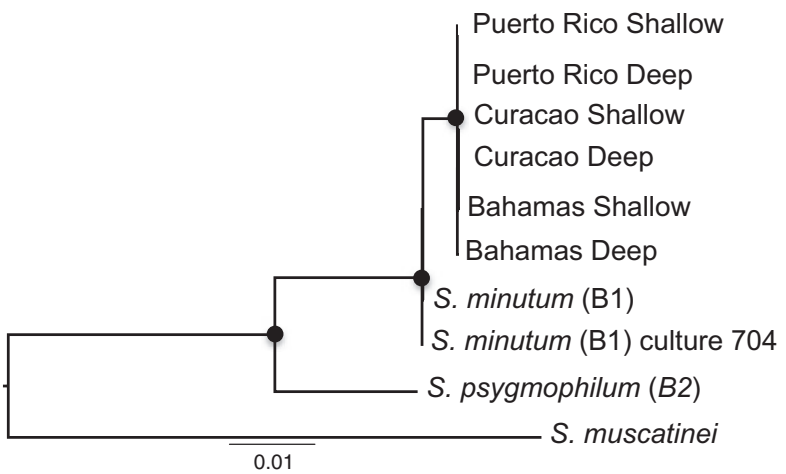

Fig. 1 Symbiodinium Clade B phylogeny based on ITS2 from colonies of Eunicea flexuosa sampled across depths and locations across the Caribbean. Black circles indicate posterior probability $>0.9$.

contained four haplotypes: two of high frequency that sorted by depth and host, and two endemic ones restricted to Bahamas Shallow and Curaçao Deep, respectively (Fig. 1B).

(a)

(b)

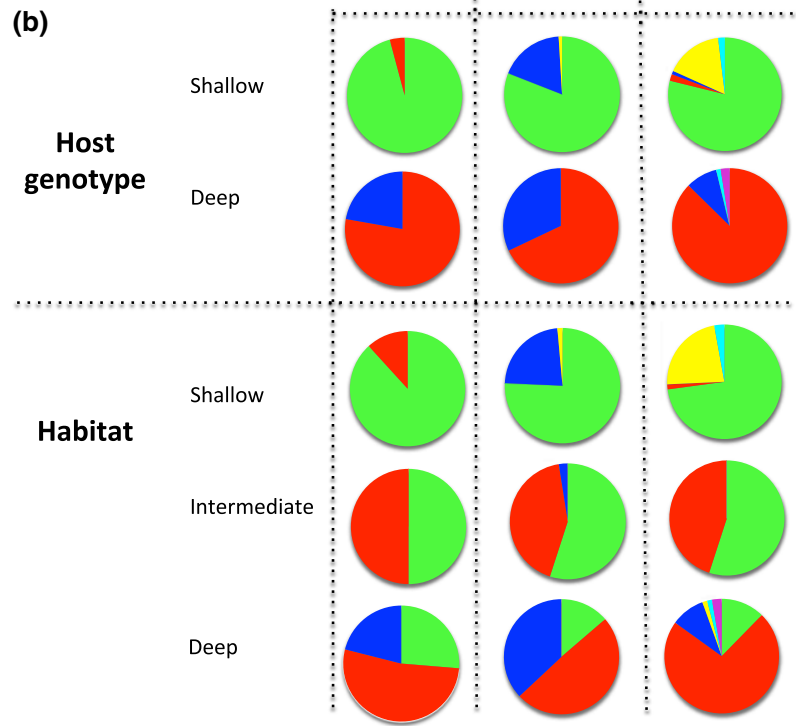

Fig. 2 Genetic diversity in Symbiodinium clade B1/B184 within E. flexuosa across the Caribbean. A) Haplotype networks for the flanking region of the microsatellite SYM15 and the chloroplast $p s b A$ and $c p 23 S$. B) Distribution of haplotypes by host genotype or depth across the Caribbean for each locus. Circles are drawn proportional to the number of individuals contained and colors signify the different haplotypes. 
Symbiodinium diversity corresponds to host lineage irrespective of depth

The AMOVA suggested that all factors (host lineage, depth and geography) significantly influenced symbiont type, with host and depth accounting for a larger proportion of the variance than geography. In a hierarchical analysis with geography, host lineage explained 93\% of the variation, while depth accounted for only $64 \%$ (Table 1), with contributions from geography becoming insignificant. Genetic variation thus is primarily divided by host lineage.

To further disentangle the role of host lineage and depth in predicting the symbiont lineage, we sampled colonies at an intermediate depth in Puerto Rico, where the two host lineages co-occur. Our results suggest that the influence of host lineage is stronger than depth: for the 40 colonies, we sampled from intermediate depth, and we found an absolute host-algal lineage association (Fig. 3). In addition, two colonies mismatched to habitats for host lineage (i.e. Eunicea flexuosa Shallow corals found in deep environments) were also mismatched for their symbiont lineage. In Curaçao, for example, where about half of the colonies in deep environments are E. flexuosa Shallow, these depth-mismatched colonies hosted Symbiodinium B1/B184 Shallow symbiont. This host-symbiont association was also evident in Puerto Rico.

\section{Symbiodinium B1/184 host-depth associated lineages} segregate further geographically

Bayesian clustering of microsatellite variation suggests two major gentic clusters of symbionts that largely correspond to the host shallow and deep lineages, respectively (Fig. 4). Both the short and the long data sets provided similar results; we present results from the full data set in the main text and results from the short data set as supplementary material (Fig. S1, Supporting information). When $\mathrm{K}$ was increased to five ( $\mathrm{K}$ with

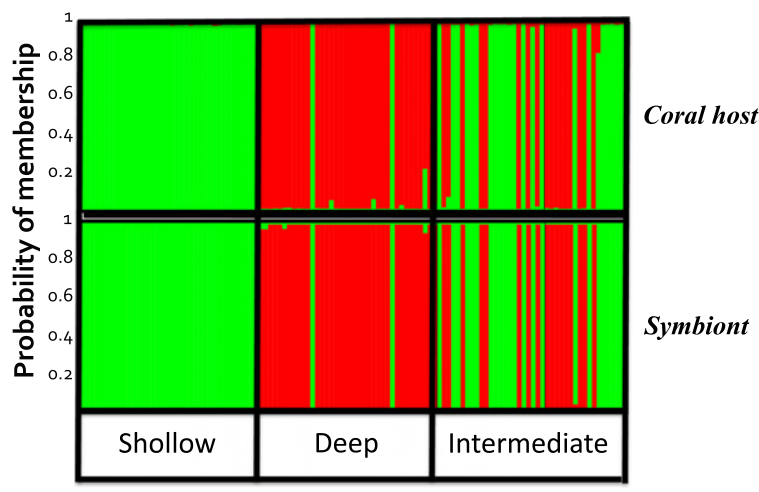

Fig. 3 STRUCTURE results $(K=2)$ for host and Symbiodinium sampled at shallow, deep and intermediate sites in Puerto Rico.

highest likelihood), geographical differentiation within each shallow/deep symbiont clusters was revealed (Fig. 4). This geographical structure within Symbiodinium contrasts with that of the host, where both deep and shallow lineages are apparently connected across the Caribbean (Prada \& Hellberg 2013).

\section{Host-Symbiodinium associations were temporally stable}

Of the 26 colonies for which pre- and post-transplantation Symbiodinium genotypic data were available, three (of 15) switched from Deep to Shallow B1/B184 $(P=0.22$, Fisher's exact test two tails) and two (of 11) from Shallow to Deep ( $P=0.24$, Fisher's exact test two tails). We also observed three (out of 26) switching to a different symbiont in the same lineage. Symbiodinium genotypes thus are generally temporally stable over a timescale of 18 months, yet not absolutely so. This within-lineage symbiont change may be due to withincolony variation in symbiont types, resulting from sampling different parts of the colony before and after transplantation, or a change in the frequencies of types already present in the colony prior to transplantation.

Table 1 Analysis of molecular variance partitioning genetic subdivision within the two Symbiodinium lineages among host lineage, depths and geography. Significant values $(P<0.05)$ are in bold

\begin{tabular}{|c|c|c|c|c|c|c|}
\hline & \multicolumn{2}{|l|}{$p s b \mathrm{~A}$} & \multicolumn{2}{|l|}{ cp23s } & \multicolumn{2}{|c|}{ Overall } \\
\hline & FST & $\%$ Variance & FST & $\%$ Variance & FST & $\%$ Variance \\
\hline Host lineage & 0.611 & 61.1 & 0.692 & 69.2 & 0.652 & 65.2 \\
\hline Depth & 0.497 & 49.7 & 0.58 & 58 & 0.539 & 53.9 \\
\hline Geography & 0.512 & 51.2 & 0.293 & 29.3 & 0.41 & 41 \\
\hline \multicolumn{7}{|c|}{ Host lineage - Geography } \\
\hline Host lineage & 0.663 & 66.3 & 0.929 & 92.9 & 0.786 & 78.6 \\
\hline Geography & 0.297 & 29.7 & 0.014 & -1.4 & 0.153 & 15.3 \\
\hline \multicolumn{7}{|c|}{ Depth - Geography } \\
\hline Depth & 0.399 & 39.9 & 0.64 & 64 & 0.51 & 51 \\
\hline Geography & 0.353 & 35.3 & 0.033 & 3.3 & 0.205 & 20.5 \\
\hline
\end{tabular}




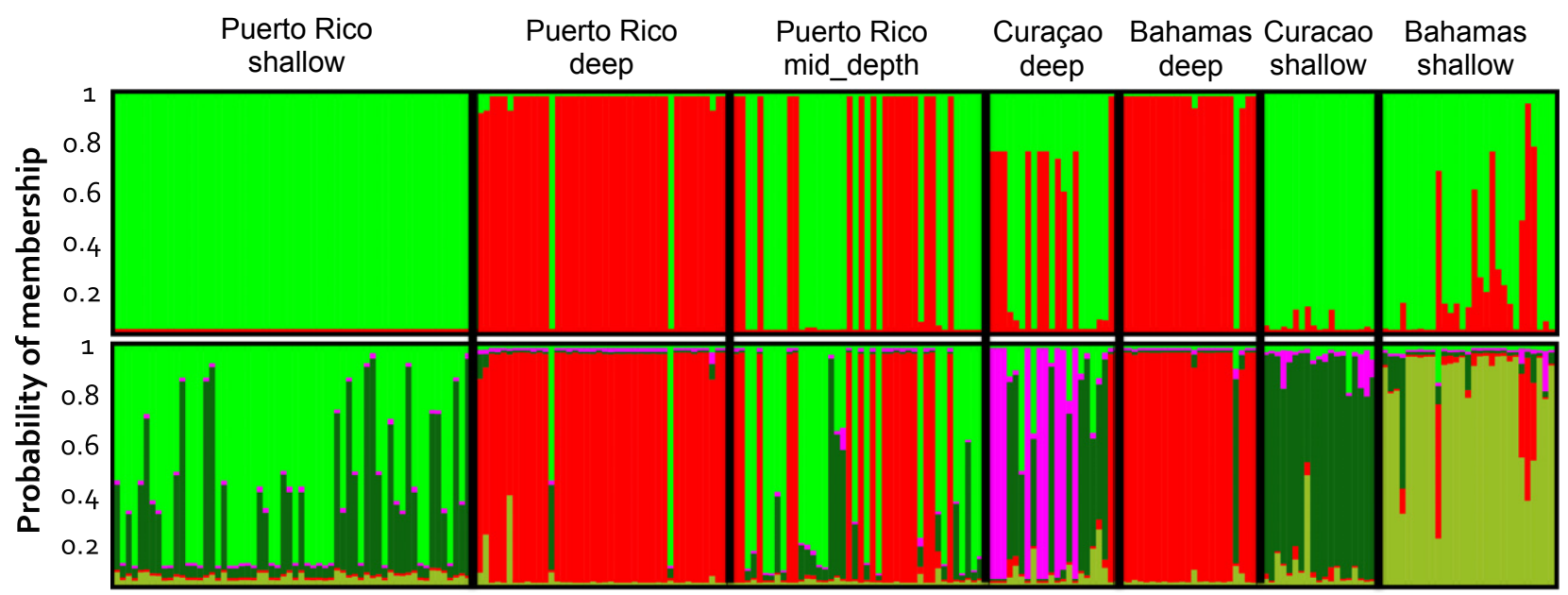

Fig. 4 Bayesian clustering for all microsatellites and all samples combined for Symbiodinium B1/184. Top panel shows $\mathrm{K}=2$ as suggested by the Evanno method and lower panel shows $\mathrm{K}=5$, $\mathrm{K}$ with the highest likelihood.

\section{Discussion}

We found two closely related, yet distinct lineages of Symbiodinium clade B that associate tightly with distinct lineages of their host Eunicea flexuosa. Although both host coral and their corresponding symbiont types are associated with different depths, surveys of hosts at intermediate depths and transplant experiments demonstrate that the effect of host identity is stronger than that of depth. Divergence within each Symbiodinium lineage largely follows a geographical pattern of segregation. The tight association between octocorals and their Symbiodinium species seen here only becomes evident when cryptic host species are recognized, revealing a holobiont (coral + symbiotic algae) unit critical for understanding adaptation at each depth or geographical locale.

\section{Cryptic species and co-evolution}

The abundance of cryptic species among both host and symbionts has led to the recognition that 'ecological specialists' may dominate coral-Symbiodinium symbioses (Finney et al. 2010). The discovery of correlated cryptic diversity in this octocoral and Symbiodinium association echoes co-evolutionary studies in terrestrial systems, in which the use of populations of both partners, along with multiple molecular markers analysed within a phylogenetic framework, have been critical to delineating symbiotic relationships. For example, in cases in which the coevolutionary status of fig wasp interactions have been questioned, finer scale genetic variation has been critical to understand the one-to-one interaction of fig trees and their pollinating wasps (Kobmoo et al. 2010). Similarly, ecotypic morphological variation in yucca plants drove the evolution of two species of pollinator moths, so that once plant cryptic variation is accounted for, the pattern of co-evolution is clear in an otherwise widespread and promiscuous plant species (Godsoe et al. 2008). Perhaps more relevant, given the tight physiological interaction reflected in coral-symbiont associations, are yeast-termite (Prillinger et al. 1996) and legume-rhizobium (Heath et al. 2012) mutualisms. In both cases, revealing cryptic variation in the yeast and bacterial symbionts has exposed dozens of species that specialize on a single host and in some cases even particular habitats within those hosts (Prillinger et al. 1996; Heath et al. 2012). In rhizobium, the genomes of both partners work in concert, and genetic variation within either species generate fitness differences to the plant (Heath et al. 2012). In a similar way, we are starting to understand how the functional genome of corals changes in predictable ways depending on the types of Symbiodinium they host (DeSalvo et al. 2010). Cryptic diversity present in the lineages of Eunicea flexuosa and Symbiodinium B1/B184 may be an ecologically relevant association, with fitness costs to both for mismatching and genomic coordination that has evolved over many generations.

Failing to recognize cryptic species in either host or symbiont may lead to an underestimation of diversity, an overestimation of the ecological and physiological ranges of individual species (i.e., one generalist instead of many specialists), and a mistaken view of how species acclimate or adapt to changing conditions. Wirshing et al. (2013), for example, did not consider cryptic species within E. flexuosa and Symbiodinium, leading to their conclusion of a flexible association between E. flexuosa and Symbiodinium B1/B184 and geographical variation within E. flexuosa. They made no note of the depth of their sampled colonies, opening the possibility that geography could be confounded with cryptic species identity. This seems likely in Panama (their most divergent population), where both lineages of E. flexuosa co-occur in shallow areas (Prada \& Hellberg 2013). Bocas del Toro 
(Panama) is an atypical Caribbean location in which local sedimentation often draws organisms typical of deep water into shallow depths. Black corals, for example, can be observed as shallow as $5 \mathrm{~m}$, whereas they occur $>20 \mathrm{~m}$ elsewhere in the Caribbean (Opresko \& Sanchez 2005). Wirshing et al. (2013) also note null alleles for all loci (except Plf67) in their Panama samples. Such a pattern would be expected if the microsatellites amplify well for the shallow lineage (for which the primers were developed), but fail for colonies of the divergent deep lineage, which are at high frequency in shallow areas in Panama. Thus, when reconsidered in the light of cryptic host species, E. flexuosa's symbiont associations are like those of other Caribbean gorgonians, in which different Symbiodinium phylotypes associate with different octocoral species (Santos et al. 2004).

Co-evolutionary patterns of divergence are not restricted to octocorals. Recent work in several brooding corals, including Agaricia (Bongaerts et al. 2013), Seriatopora (Bongaerts et al. 2010) and Madracis (Frade et al. 2008), suggests that species or genetically differentiated populations have co-evolved with their algal symbionts. Specific coral-symbiont associations seem more prevalent in brooding scleractinians in which Symbiodinium are transmitted vertically. The Symbiodinium - E. flexuosa tight association is remarkable and similar to that in Orbicella species (Thornhill et al. 2014) because specialization proceeded in a broadcasting species in the absence of direct vertical transmission and in a system in which larvae can travel large distances $(>10 \mathrm{~km})$ (Prada \& Hellberg 2013; Wirshing et al. 2013) and only acquire symbionts once the larvae have settled and metamorphosed into a polyp (Poland et al. 2013).

The coral-symbiont association need not be viewed as a strict dichotomy of flexibility or species specificity. While specificity is common in octocorals (Santos et al. 2004; Goulet 2006), some scleractinian corals acquire various Symbiodinium phylotypes over ecological scales (Rowan et al. 1997; LaJeunesse et al. 2009). However, such apparent flexibility contains finer specificity (Finney et al. 2010). Orbicella faveolata, for example, associates with Symbiodinium from clades A, B, C and D, but only with specific phylotypes within those four clades, especially within clade C (Thornhill et al. 2014). Such mixed patterns of flexibility at macroevolutionary timescales and specificity at microevolutionary scales beg for more studies to understand the evolution of symbiosis in anthozoans.

\section{Depth, photobiology and ecological speciation}

Diversity in Symbiodinium has often been associated with habitat heterogeneity. In a landmark study, Rowan et al. (1997) showed how subtle changes in light availability within single coral colonies can segregate Symbiodinium genetic variation. More recent work suggests that depth, often correlated with light, underlies Symbiodinium variation across habitats (Sampayo et al. 2008; Kirk et al. 2009; Finney et al. 2010; Lesser et al. 2010). In these heterogeneous environments, different host-symbiont combinations cause differences in survivorship and growth for the holobiont (Berkelmans \& van Oppen 2006; Sampayo et al. 2007). Segregation in Symbiodinium types in Eunicea flexuosa is associated with a transition in depth, raising the likelihood that different host-symbiont combinations perform better at their native depths (Sampayo et al. 2007).

Studies of the photobiology of Symbiodinium suggest that different species have different capacities and perform better at different light conditions, such those that vary with depth (Iglesias-Prieto \& Trench 1994). B1 species (as in E. flexuosa), for example, show different biophysical properties, such active fluorescence and photosystem-specific signatures (Robison \& Warner 2006; Hennige et al. 2008, 2011). Moreover, Symbiodinium species segregated by depth have different light optima and enhanced capacity (i.e., photosystem pressure) at native depths, even if these depth-segregated species are reciprocally transplanted (Iglesias-Prieto et al. 2004). It is thus likely that the enhanced survivorship of E. flexuosa to each habitat is partly due to its tight association with a specific Symbiodinium type.

In E. flexuosa, the formation and maintenance of the two lineages appear to have been promoted by immigrant inviability (Prada \& Hellberg 2013), which occurs when locally adapted populations migrate to suboptimal environments, increasing mortality of these maladapted individuals (Nosil et al. 2005). For both E. flexuosa and Symbiodinium, their partner becomes another 'environment'. Immigrant inviability can thus act in two ways. First, it can act against individuals with the 'wrong' hostsymbiont coupling. Next, even for pairs with the 'right' host-symbiont coupling, inviability will sort holobionts by depth. Unlike brooders, in which most host-symbiont specialization has been observed, E. flexuosa acquires its Symbiodinium horizontally (from the surrounding environment upon settlement, rather than via its parent), increasing the potential for immigrant inviability.

\section{Geographical variation and phylogeography}

Symbiodinium in Eunicea flexuosa shows patterns of geographical differentiation within each shallow and deep lineage, along with the segregation by host species and depth. Geographical differentiation is pervasive in Symbiodinium, and most studies have reported geographical differentiation often (as here) at scales smaller than their host (Andras et al. 2011). For example, Andras et al. (2011) found that populations of Symbiodinium are similar between Puerto Rico and the Bahamas but 
different from those in Curaçao. We found the same pattern for Symbiodinium associated with E. flexuosa Deep, but a full segregation across localities for the shallow lineage. The higher partition across geography by the shallow lineage suggests that even at small depth contours, physical and biological processes vary in the Caribbean, uniquely altering connectivity patterns of populations at different depths.

\section{Conclusion}

The high specificity between genetically distinct populations of Symbiodinium with different Eunicea flexuosa lineages suggests a pattern of co-evolution such that this coupling defines the fitness of the holobiont in different environments. Other symbiotic or parasitic partners, such as bacteria, fungi and viruses, may further shape survivorship and reproduction of the holobiont. These species interactions may influence evolutionary patterns and, when coupled with ecological factors such depth, may play a role in speciation. During ecological speciation, species interactions may reinforce prezygotic isolation by preventing the establishment of maladapted coral-symbiont-depth combinations, each of these combinations being a potential link at which immigrant inviability can operate. Knowlton (1993) highlighted the vast diversity of sibling species in the sea. We are now beginning to understand how marine diversity segregated along ecological factors like depth creates complexes of codiversifying species and increases diversity in the sea.

\section{Acknowledgements}

We thank the Department of Marine Sciences of the University of Puerto Rico, Mayagüez for assistance during the transplant experiment (R. Appeldorn) (NOAA- NA06NOS4780190). Comments from T. Lajeunesse and M. Debiasse improved this study. We thank the governments of Puerto Rico, Curaçao and The Bahamas for granting collecting permits. We thank H. Lasker for support during collections in the Bahamas, along with the crew of the R/V Walton Smith of the University of Miami. We also thank M. Vermeij and CARMABI, Curaçao. This work was supported by a Lerner Gray Grant from the American Museum of Natural History, a Rosemary Grant Research Award from the Society for the Study of Evolution and a Research Award from the Society of Systematic Biologists to CP. CP has been supported by Huel Perkins and Dissertation Year fellowships from LSU. Partial funds came from the LSUDepartment of Biological Sciences and DDIG-NSF awarded to CP and MEH (DEB-1311579) and NSF grants to IB Baums and MEH (OCE-0550270) and MAC (OCE-0926822).

\section{References}

Abrego D, Ulstrup KE, Willis BL, van Oppen MJH (2008) Species-specific interactions between algal endosymbionts and coral hosts define their bleaching response to heat and light stress. Proceedings of the Royal Society of London, Series B: Biological Sciences, 275, 2273-2282.

Andras JP, Kirk NL, Coffroth MA, Harvell CD (2009) Isolation and characterization of microsatellite loci in Symbiodinium B1/ B184, the dinoflagellate symbiont of the Caribbean sea fan coral, Gorgonia ventalina. Molecular Ecology Notes, 9, 989-993.

Andras JP, Kirk NL, Harvell CD (2011) Range-wide population genetic structure of Symbiodinium associated with the Caribbean Sea fan coral, Gorgonia ventalina. Molecular Ecology, 20, 2525-2542.

Baker A (1999) Reef corals bleach to survive change. Nature, 411, 765-766.

Bayer FM (1961) The Shallow-Water Octocorallia of the West Indian Region. A Manual for marine biologists. Martinus Nijhoff, The Hague.

Beiring EA, Lasker HR (2000) Egg production by colonies of a gorgonian coral. Marine Ecology Progress Series, 196, 169-177.

Berkelmans R, van Oppen MJH (2006) The role of zooxanthellae in the thermal tolerance of corals: a 'nugget of hope' for coral reefs in an era of climate change. Proceedings of the Royal Society of London, Series B: Biological Sciences, 273, 2305-2312.

Bongaerts P, Riginos C, Ridgway T et al. (2010) Genetic divergence across habitats in the widespread coral Seriatopora hystrix and its associated Symbiodinium. PLoS ONE, 5, 1871.

Bongaerts P, Frade PR, Ogier JJ et al. (2013) Sharing the slope: depth partitioning of agariciid corals and associated Symbiodinium across shallow and mesophotic habitats $(2-60 \mathrm{~m})$ on a Caribbean reef. BMC Evolutionary Biology, 13, 205.

Chen C, Wang A, Fang L, Yang Y (2005) Fluctuating algal symbiont communities in Acropora palifera (Scleractinia: Acroporidae) from Taiwan. Marine Ecology Progress Series, 295, 113-121.

Clement M, Posada D, Crandall K (2000) TCS: a computer program to estimate gene genealogies. Molecular Ecology, 9, 1657-1660.

Coffroth MA, Santos SR (2005) Genetic diversity in Symbiodinium. Protist, 156, 19-34.

Coffroth MA, Lasker HR, Diamond ME, Bruenn JA, Bermingham E (1992) DNA fingerprints of a gorgonian coral: a method for detecting clonal structure in a vegetative species. Marine Biology, 114, 317-325.

Coffroth MA, Santos SR, Goulet TL (2001) Early ontogenetic expression of specificity in a cnidarian-algal symbiosis. Marine Ecology Progress Series, 222, 85-96.

Colombo-Pallotta MF, Rodríguez-Román A, Iglesias-Prieto $\mathrm{R}$ (2010) Calcification in bleached and unbleached Montastraea faveolata: evaluating the role of oxygen and glycerol. Coral Reefs, 29, 899-907.

Davy SK, Allemand D, Weis VM (2012) Cell biology of cnidarian-dinoflagellate symbiosis. Microbiology and Molecular Biology Reviews, 76, 229-261.

DeSalvo MK, Sunagawa S, Fisher PL, Voolstra CR, Iglesias-Prieto R, Medina M (2010) Coral host transcriptomic states are correlated with Symbiodinium genotypes. Molecular Ecology, 19, 1174-1186.

Drummond AJ, Ashton B, Cheung M et al. (2009) Geneious v4.5.4. Available from http://www.geneious.com.

Earl D, vonHoldt E (2011) STRUCTURE HARVESTER: a website and program for visualizing STRUCTURE output and implementing the Evanno method. Conservation Genetics Resources, 4, 359-361. 
Evanno G, Regnaut S, Goudet J (2005) Detecting the number of clusters of individuals using the software STRUCTURE: a simulation study. Molecular Ecology, 14, 2611-2620.

Finney JC, Pettay T, Sampayo EM, Warner ME, Oxenford HA, LaJeunesse TC (2010) The relative significance of host-habitat, depth, and geography on the ecology, endemism, and speciation of coral endosymbionts in the genus Symbiodinium. Microbial Ecology, 60, 250-263.

Frade PR, De Jongh F, Vermeulen F, Van Bleijswijk J, Bak RPM (2008) Variation in symbiont distribution between closely related coral species over large depth ranges. Molecular Ecology, 17, 691-703.

Godsoe W, Yoder JB, Smith CP, Pellmyr O (2008) Coevolution and diversification in the Joshua tree yucca moth mutualism. American Naturalist, 171, 816-823.

Goulet TL (2006) Most corals may not change their symbionts. Marine Ecology Progress Series, 321, 1-7.

Goulet TL, Coffroth MA (2003) Genetic composition of zooxanthellae between and within colonies of the octocoral Plexaura kuna, based on small subunit rDNA and multilocus DNA fingerprinting. Marine Biology, 142, 233-239.

Goulet TL, Simmons C, Goulet D (2008) Worldwide biogeography of Symbiodinium in tropical octocorals. Marine Ecology Progress Series, 355, 45-58.

Heath KD, Burke PV, Stinchcombe JR (2012) Coevolutionary genetic variation in the legume-rhizobium transcriptome. Molecular Ecology, 21, 4735-4747.

Hennige SJ, Suggett DJ, Warner ME, McDougall KE, Smith DJ (2008) Unravelling coral photoacclimation: Symbiodinium strategy and host modification. In: Proceedings of the 11th International Coral Reef Symposium (eds Bruckner AW, Roberts G), pp. 7-11, Ft. Lauderdale, Florida.

Hennige SJ, McGinley M, Grottoli AG, Warner ME (2011) Photoinhibition of Symbiodinium spp. within the reef corals Montastraea faveolata and Porites astreoides: implications for coral bleaching. Marine Biology, 158, 2515-2526.

Huelsenbeck JP, Ronquist F (2001) MrBayes: Bayesian inference of phylogenetic trees. Bioinformatics, 17, 754-755.

Iglesias-Prieto R, Trench RK (1994) Adaptation and acclimation to irradiance in symbiotic dinoflagellates I. Response of the photosynthetic unit to changes in photon flux density. Marine Ecology Progress Series, 113, 163-175.

Iglesias-Prieto R, Beltrán VH, LaJeunesse TC, Reyes-Bonilla H, Thomé PE (2004) Different algal symbionts explain the vertical distribution of dominant reef corals in the eastern Pacific. Proceedings of the Royal Society of London, Series B: Biological Sciences, 271, 1757-1763.

Jakobsson M, Rosenberg N (2007) CLUMPP: a cluster matching and permutation program for dealing with label switching and multimodality in analysis of population structure. Bioinformatics, 23, 1801.

Kahng S, Benayahu B, Lasker HR (2011) Sexual reproduction in octocoral. Marine Ecology Progress Series, 443, 265-283.

Kim E, Lasker HR, Coffroth MA, Kim K (2004) Morphological and genetic variation across reef habitats in a broadcastspawning octocoral. Hydrobiologia, 530/531, 423-432.

Kirk NL, Andras JP, Harvell CD, Santos SR, Coffroth MA (2009) Population structure of Symbiodinium sp. associated with the common sea fan, Gorgonia ventalina, in the Florida Keys across distance, depth, and time. Marine Biology, 156, 1609-1623.
Knowlton N (1993) Sibling species in the sea. Annual Review of Ecology, Evolution and Systematics, 24, 189-216.

Kobmoo M, Hossert-McKey M, Rasplus JY, Kjellberg F (2010) Ficus racemosa is pollinated by a single population of a single agaonid wasp species in continental South-East Asia. Molecular Ecology, 19, 2700-2712.

LaJeunesse TC (2002) Diversity and community structure of symbiotic dinoflagellates from Caribbean coral reefs. Marine Biology, 141, 387-400.

LaJeunesse TC (2005) "Species" radiations of symbiotic dinoflagellates in the Atlantic and Indo-Pacific since the Miocene-Pliocene transition. Molecular Biology and Evolution, 22, 570-581.

LaJeunesse TC, Thornhill DJ, Cox EF, Stanton FG, Fitt WK, Schmidt GW (2004) High diversity and host specificity observed among symbiotic dinoflagellates in reef coral communities from Hawaii. Coral Reefs, 23, 596-603.

LaJeunesse TC, Smith RT, Finney JC, Oxenford HA (2009) Outbreak and persistence of opportunistic symbiotic dinoflagellates during the 2005 Caribbean mass coral 'bleaching' event. Proceedings of the Royal Society of London, Series B: Biological Sciences, 276, 4139-4148.

LaJeunesse TC, Pettay DT, Sampayo EM et al. (2010) Longstanding environmental conditions, geographic isolation and host-symbiont specificity influence the relative ecological dominance and genetic diversification of coral endosymbionts in the genus Symbiodinium. Journal of Biogeography, $37,785-800$.

LaJeunesse TC, Parkinson JE, Reimer JD (2012) A geneticsbased description of Symbiodinium minutum sp. nov. and S. psygmophilum sp. nov. (Dinophyceae), two dinoflagellates symbiotic with Cnidaria. Journal of Phycology, 48, 1380-1391.

Lesser MP, Ojimi M, Gates R, Stat M, Slattery M, Grottoli AG (2010) Photoacclimatization by the Coral Montastraea cavernosa in the Mesophotic Zone: Light, Food, and Genetics. Ecology, 91, 990-1003.

Little AF, van Oppen MJH, Willis BF (2004) Flexibility in algal endosymbioses shapes growth in reef corals. Science, 304, 1492-1494.

Magalon H, Baudry E, Huste A, Adjeroud M, Veuille M (2006) High genetic diversity of the symbiotic dinoflagellates in the coral Pocillopora meandrina from the South Pacific. Marine Biology, 148, 913-922.

Meirmans PG, Van Tienderen PH (2004) GENOTYPE and GENODIVE: two programs for the analysis of genetic diversity of asexual organisms. Molecular Ecology Notes, 4, 792-794.

Mieog J, van Oppen M, Cantin N, Stam W, Olsen J (2007) Realtime PCR reveals a high incidence of clade D at low levels in four scleractinian corals across the Great Barrier Reef: implications for symbiont shuffling. Coral Reefs, 26, 449-457.

Moore RB, Ferguson KM, Loh WKW, Hoegh-Guldberg O, Carter DA (2003) Highly organized structure in the non-coding region of the psbA minicircle from clade C Symbiodinium. International Journal of Systematic and Evolutionary Microbiology, 53, 1725-1734.

Muscatine L, McCloskey LR, Marian RE (1981) Estimating the daily contribution of carbon from zooxanthellae to coral animal respiration. Limnology and Oceanography, 26, 601-611.

Muscatine L, Falkowski PG, Dubinsky Z (1983) Carbon budgets in symbiotic associations. In: Endocytobiology II. Intracel- 
lular Space as Oligogenetic Ecosystem(eds Schenk HEA \& Schwemmler W), pp. 649-673. Walter de Gruyter, Berlin and New York

Muscatine L, Falkowski PG, Porter JW, Dubinsky Z (1984) Fate of photosynthetic fixed carbon in light- and shadeadapted colonies of the symbiotic coral Stylophora pistillata. Proceedings of the Royal Society of London, Series B: Biological Sciences, 222, 181-202.

Nylander JAA (2004) Mrmodeltest Version 2.2. Program distributed by the author. Evolutionary Biology Centre, Uppsala University.

Nosil P, Vines T, Funk D (2005) Perspective: reproductive isolation caused by natural selection against immigrants from divergent habitats. Evolution, 59, 705-719.

Opresko DM, Sanchez JA (2005) Caribbean shallow-water black corals (Cnidaria: Anthozoa: Antipatharia). Caribbean Journal of Science, 41, 492-507.

Pettay DT, LaJeunesse TC (2007) Microsatellites from clade B Symbiodinium spp. specialized for Caribbean corals in the genus Madracis. Molecular Ecology Notes, 7, 1271-1274.

Pettay DT, Wham DC, Pinzón JH, LaJeunesse TC (2011) Genotypic diversity and spatial-temporal distribution of Symbiodinium clones in an abundant reef coral. Molecular Ecology, 20, 5197-5212.

Pinzon JH, LaJeunesse TC (2011) Species delimitation of common reef corals in the genus Pocillopora using nucleotide sequence phylogenies, population genetics, and symbiosis ecology. Molecular Ecology, 20, 311-325.

Pochon X, Gates RD (2010) A new Symbiodinium clade (Dinophyceae) from soritid foraminifera in Hawai'i. Molecular Phylogenetics and Evolution, 56, 492-497.

Poland DM, Mansfield JM, Kirk NL et al. (2013) Annual and habitat variation in Symbiodinium acquisition by juvenile Briareum asbestinum (Cnidaria: Octocorallia). Marine Ecology Progress Series, 476, 23-37.

Prada C, Hellberg ME (2013) Long pre-reproductive selection and divergence by depth in a Caribbean candelabrum coral. Proceedings of the National Academy of Sciences of the USA, 119, 53-60.

Prada C, Schizas NV, Yoshioka PM (2008) Phenotypic plasticity or speciation? A case from a clonal marine organism. BMC Evolutionary Biology, 8, 47.

Prillinger H, Messner R, König H et al. (1996) Yeasts associated with termites: a phenotypic and genotypic characterization and use of coevolution for dating evolutionary radiations in asco- and basidiomycetes. Systematic and Applied Microbiology, 19, 265-283.

Pritchard JK, Stephens M, Donnelly P (2000) Inference of population structure using multilocus genotype data. Genetics, 155, 945-959.

Robison JD, Warner ME (2006) Differential impacts of photoacclimation and thermal stress on the photobiology of four different phylotypes of Symbiodinium (pyrrhophyta) 1. Journal of Phycology, 42, 568-579.

Rosenberg NA (2004) DISTRUCT: a program for the graphical display of population structure. Molecular Ecology Notes, 4, 137-138.

Rowan R (2004) Coral bleaching: thermal adaptation in reef coral symbionts. Nature, 430, 742.

Rowan R, Knowlton N (1995) Intraspecific diversity and ecological zonation in coral-algal symbiosis. Proceedings of the National Academy of Sciences of the USA, 92, 2850-2853.
Rowan R, Powers DA (1992) Ribosomal RNA sequences and the diversity of symbiotic dinoflagellates (zooxanthellae). Proceedings of the National Academy of Sciences of the USA, 89, 36393643.

Rowan R, Knowlton N, Baker A, Jara J (1997) Landscape ecology of algal symbionts creates variation in episodes of coral bleaching. Nature, 388, 265-269.

Sampayo E, Franceschinis L, Hoegh-Guldberg O, Dove S (2007) Niche partitioning of closely related symbiotic dinoflagellates. Molecular Ecology, 16, 3721-3733.

Sampayo EM, Ridgway T, Bongaerts P, Hoegh-Guldberg O (2008) Bleaching susceptibility and mortality of corals are determined by fine-scale differences in symbiont type. Proceedings of the National Academy of Sciences of the USA, 105, 10444-10449.

Santos SR, Taylor DJ, Kinzie RA, Hidaka M, Sakai K, Coffroth MA (2002) Molecular phylogeny of symbiotic dinoflagellates inferred from partial chloroplast large subunit 23srDNA sequences. Molecular Phylogeetics and Evolution, 23, 97-111.

Santos S, Gutierrez-Rodriguez C, Coffroth M (2003a) Phylogenetic identification of symbiotic dinoflagellates via length heteroplasmy in domain $\mathrm{V}$ of chloroplast large subunit (cp23s)-rDNA sequences. Marine Biotechnology, 5, 130-140.

Santos SR, Gutierrez-Rodriguez C, Lasker HR, Coffroth MA (2003b) Symbiodinium sp. associations in the gorgonian Pseudopterogorgia elisabethae in the Bahamas: high levels of genetic variability and population structure in symbiotic dinoflagellates. Marine Biology, 143, 111-120.

Santos SR, Shearer TL, Hannes AR, Coffroth MA (2004) Finescale diversity and specificity in the most prevalent lineage of symbiotic dinoflagellates (Symbiodinium, Dinophyceae) of the Caribbean. Molecular Ecology, 13, 459-469.

Silverstein RN, Correa A, Baker A (2012) Specificity is rarely absolute in coral-algal symbiosis: implications for coral response to climate change. Proceedings of the Royal Society of London, Series B: Biological Sciences, 279, 2609-2618.

Templeton AR, Crandall KA, Sing CF (1992) A cladistic analysis of phenotypic associations with haplotypes inferred from restriction endonuclease mapping with DNA sequence data. III. Cladogram estimation. Genetics, 132, 619-633.

Thornhill DJ, Xiang Y, Fitt WK, Santos SR (2009) Reef endemism, host specificity and temporal stability in populations of symbiotic dinoflagellates from two ecologically dominant Caribbean corals. PLOS ONE, 4, e6262.

Thornhill DJ, Xiang Y, Pettay PM, Zhong M, Santos SR (2013) Population genetic data of a model symbiotic cnidarian system reveal remarkable symbiotic specificity and vectored introductions across ocean basins. Molecular Ecology, 22, 4499-4515.

Thornhill DJ, Lewis AM, Wham DC, LaJeunesse TC (2014) Host-specialist lineages dominate the adaptive radiation of reef coral endosymbionts. Evolution, 68, 352-367.

Voolstra CR, Schwarz JA, Schnetzer J et al. (2009) The host transcriptome remains unaltered during the establishment of coral-algal symbioses. Molecular Ecology, 18, 18231833.

Wirshing H, Feldhein KA, Baker AW (2013) Vectored dispersal of Symbiodinium by larvae of a Caribbean gorgonian octocoral. Molecular Ecology, 22, 4413-4432. 
C.P., M.A.C. and M.E.H. conceived the project. C.P. and D.M.B. did transplant experiment, collections and DNA sequencing. D.J.V., S.E.M., S.A.F. and M.A.C. did cp23s analysis and microsatellite genotyping. C.P. did all analysis. C.P. and M.E.H. wrote the manuscript. All authors commented and agreed on the manuscript.

\section{Data accessibility}

Sequence data is available from GenBank. Accession nos for Eunicea flexuosa are: KC310499-KC310687 and
KC333998-KC335131. Accession nos for Symbiodinium B1/B184 are: ITS2 (KJ780833 - KJ780838), cp23s (KJ780839 - KJ781021) and psbA (KJ781022 - KJ781204).

The short and the long microsatellite data sets along with alignments for all sequences are available through Dryad (doi:10.5061/dryad.ns34t).

\section{Supporting information}

Additional supporting information may be found in the online version of this article.

Fig. S1 Bayesian clustering for the short dataset for all microsatellites for Symbiodinium B1/184. 